Article

\title{
High-Energy, Multicolor Femtosecond Pulses from the Deep Ultraviolet to the Near Infrared Generated in a Hydrogen-Filled Gas Cell and Hollow Fiber
}

\author{
Kazuya Motoyoshi ${ }^{1}$, Yuichiro Kida ${ }^{1, *}$ and Totaro Imasaka ${ }^{1,2}$ \\ 1 Department of Applied Chemistry, Graduate School of Engineering, Kyushu University, \\ 744 Motooka, Nishi-ku, Fukuoka 819-0395, Japan; \\ E-Mails: motoyoshi.kazuya.555@s.kyushu-u.ac.jp (K.M.); imasaka@cstf.kyushu-u.ac.jp (T.I.) \\ 2 Division of Optoelectronics and Photonics, Center for Future Chemistry, Kyushu University, \\ 744 Motooka, Nishi-ku, Fukuoka 819-0395, Japan \\ * Author to whom correspondence should be addressed; E-Mail: y-kida@cstf.kyushu-u.ac.jp; \\ Tel.: +81-92-802-2886; Fax: +81-92-802-2888.
}

Received: 4 April 2014; in revised form: 29 May 2014 / Accepted: 13 June 2014 /

Published: 1 July 2014

\begin{abstract}
We investigate four-wave mixing in hydrogen gas using a gas cell and a hollow fiber for the generation of high-energy, multicolor femtosecond (fs) optical pulses. Both a hydrogen-filled gas cell and hollow fiber lead to the generation of multicolor fs pulses in a broad spectral range from the deep ultraviolet to the near infrared. However, there is a difference in the energy distribution of the multicolor emission between the gas cell and the hollow fiber. The hydrogen-filled gas cell generates visible pulses with higher energies than the pulses created by the hollow fiber. We have generated visible pulses with energies of several tens of microjoules. The hydrogen-filled hollow fiber, on the other hand, generates ultraviolet pulses with energies of a few microjoules, which are higher than the energies of the ultraviolet pulses generated in the gas cell. In both schemes, the spectral width of each emission line supports a transform-limited pulse duration shorter than $15 \mathrm{fs}$. Four-wave mixing in hydrogen gas therefore can be used for the development of a light source that emits sub-20 fs multicolor pulses in a wavelength region from the deep ultraviolet to the near infrared with microjoule pulse energies.
\end{abstract}

Keywords: multicolor laser; ultrashort optical pulses; four-wave mixing; high-order sideband generation 


\section{Introduction}

Four-wave mixing (FWM) has been investigated and used in the past few decades to generate multicolor laser emission in various wavelength regions. In 1981, more than 40 laser emission lines spanning from the deep ultraviolet (DUV) to the near infrared (NIR) were generated by focusing a two-color nanosecond pulse into hydrogen gas [1]. The generation of multicolor laser emission via FWM has hitherto been extensively studied in deuterium and hydrogen gases [2-5] and extremely short optical pulses with durations shorter than 2 fs have been generated [3,5] by Fourier synthesis of the emission lines [6,7].

The generation of multicolor laser emission via FWM has also been investigated in the femtosecond (fs) regime [8-21]. Resonant and non-resonant FWM has been investigated in bulk media [11,13,15-17], leading to multicolor laser emission with a pulse energy of about 1 microjoule [17]. By employing the pre-compression technique, multicolor emission with short pulse durations less than 20 fs has been demonstrated [16]. Such short multicolor pulses can be used in ultrafast spectroscopy [22-24], as well as in nonlinear optical microscopy [25]. The efficiency of the multicolor generation can be improved by more than one order of magnitude using gaseous media rather than bulk media. In addition, the spectral range from the DUV to the NIR has been covered by employing Raman active gases as the nonlinear media for FWM [9,10,12,14,20,21]. When pumping with short fs pulses with high intensities, self-phase modulation (SPM) and cross-phase modulation (XPM) are simultaneously induced, which results in a broad spectral width for each multicolor emission. The broad spectral width is advantageous for generating a short optical pulse after adequate dispersion compensation. The maximum spectral width of each multicolor emission is limited by the frequency separation between the center frequencies of the adjacent multicolor emission, which is determined by the Raman shift of the medium used. When using the vibrational transition of molecular hydrogen with a large Raman shift frequency of $4155 \mathrm{~cm}^{-1}$ [26], it is possible to generate multicolor fs pulses with spectral widths supporting transform-limited pulse durations of about $10 \mathrm{fs}$. Multicolor generation via the vibrational transition has been investigated both in a hydrogen-filled hollow fiber using two-color fs pump pulses at 800 and $600 \mathrm{~nm}$ [12] and in a hydrogen-filled gas cell using shorter two-color NIR pump pulses emitting at 800 and $1200 \mathrm{~nm}$ [20]. These investigations have reported multicolor laser emission covering from the DUV to NIR. The use of a hollow fiber [12] and gas cell [20], however, has been investigated under independent experimental conditions. Therefore, a quantitative discussion of the efficiency of multicolor generation and the characteristics of the pulses has been hindered. Information related to the pulse energy of each multicolor emission has not been reported to date; these data are important for evaluating the multicolor laser pulses.

We investigate here two approaches of FWM in a hydrogen-filled gas cell and a hydrogen-filled hollow fiber. We compare the results to analyze laser sources of multicolor fs pulses. By maintaining the experimental parameters at a constant - except for the gas pressure, which has been optimized in each experiment - the two schemes can be directly compared with one other. We find that the gas-filled hollow fiber with a longer propagation distance than that of the gas cell does not always lead to higher efficiencies in the multicolor generation. The gas cell gives rise to visible multicolor pulses with higher pulse energies than those generated in the hollow fiber. On the other hand, the latter produces higher-energy multicolor pulses in the ultraviolet range. 


\section{Experimental Section}

Figure 1 shows the experimental setup. A part of the NIR pulse emerging from a Ti:sapphire regenerative amplifier $(800 \mathrm{~nm}, 35 \mathrm{fs}, 4 \mathrm{~mJ}, 1 \mathrm{kHz}$, Legend Elite-USP, Coherent Inc., Santa Clara, CA, USA) was used as the pump source for an optical parametric amplifier (OPA, OPerASolo, Coherent Inc., Santa Clara, CA, USA). The OPA generated a NIR pulse at $1200 \mathrm{~nm}$, whose frequency separation from the Ti:sapphire amplifier $(800 \mathrm{~nm})$ was adjusted to the vibrational Raman shift frequency of molecular hydrogen $\left(4155 \mathrm{~cm}^{-1}\right)$. The output of the OPA (pump 1; P1) was spatially combined with the remaining part of the output beam of the regenerative amplifier at $800 \mathrm{~nm}$ (pump 2; $\mathrm{P} 2$ ). The beam was focused with an off-axis parabolic mirror (with a focal length of $655 \mathrm{~mm}$ ) into a gas cell filled with hydrogen or a fused-silica hollow fiber (core diameter, $320 \mu \mathrm{m}$; length, $600 \mathrm{~mm}$ ) placed inside a gas cell (length, $1 \mathrm{~m}$ ) filled with hydrogen (hereafter denoted as a hollow fiber chamber). The core diameter of the hollow fiber of $320 \mu \mathrm{m}$ was chosen for keeping the same focusing condition as for the gas cell, and the fiber length of $600 \mathrm{~mm}$ was the maximum possible length for the hollow fiber chamber. The gas cell and the hollow fiber chamber were equipped with 0.5 -mm-thick windows made of fused silica. The time delay between the two pulses (P1 and P2) was optimized to obtain the highest energy in the highest-order anti-Stokes Raman emission generated at a gas pressure of $0.4 \mathrm{~atm}$. The spectra of the output beams from the gas cell and hollow fiber chamber were measured using a multichannel spectrometer (Maya2000pro, Ocean Optics, Dunedin, FL, USA). The spectral response was calibrated using a deuterium-halogen light source over a wavelength range of 220-900 nm.

Figure 1. The experimental setup. GM, gold mirror; SM, silver mirror; DM, dichroic mirror; PM, off-axis parabolic mirror; BS, beam splitter; DMM, dielectric multi-layer mirror; VND, variable neutral density filter.

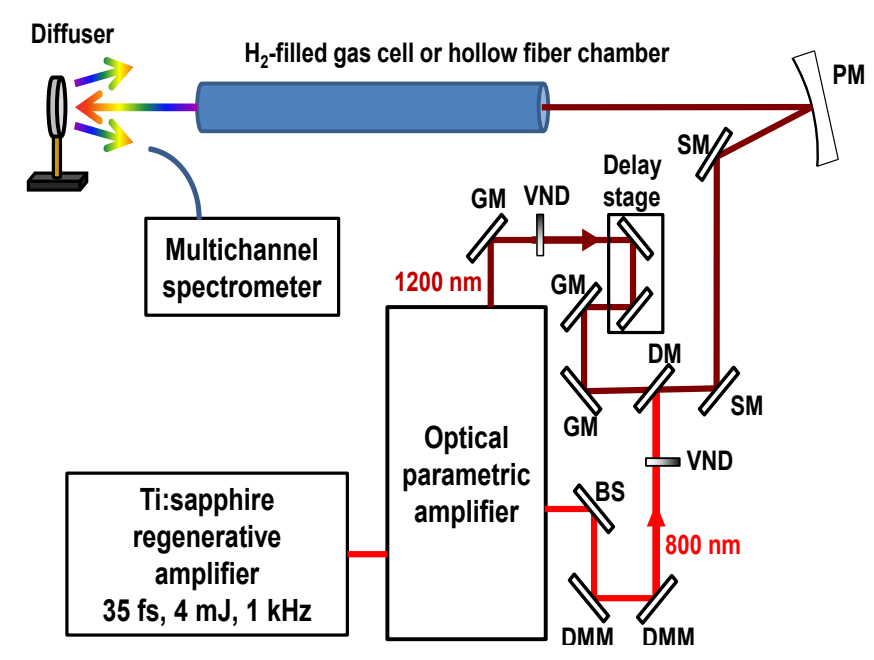

\section{Results and Discussion}

\subsection{Generation of the Multicolor Emission in the Gas Cell and the Gas-Filled Hollow Fiber}

When we used a hydrogen-filled gas cell, the FWM efficiently occurred in the vicinity of the foci at which the input beams have high intensities. The confocal parameter $\left(2 \pi W_{0}^{2} / \lambda\right)$ is a measure of the interaction length, where $W_{0}$ and $\lambda$ are the beam waist radius and the wavelength of the laser beam, 
respectively. From the measured focal beam diameters of P1 and P2-300 $\mu \mathrm{m}$ and $200 \mu \mathrm{m}$ - the confocal parameters of the beams were calculated. The value for P2 was $80 \mathrm{~mm}$, which was shorter than that of P1 $(120 \mathrm{~mm})$. The interaction length for the gas cell was, therefore, estimated to be approximately $80 \mathrm{~mm}$, which was shorter than that of the hydrogen-filled hollow fiber.

The energies of the input and output pulses measured in front of the evacuated gas cell and hollow fiber chamber are listed in Table 1. From these values, the transmittance of the gas cell was calculated to be $90 \%$ for both $\mathrm{P} 1$ and $\mathrm{P} 2$. This value is in good agreement with the value calculated from Fresnel losses on the surfaces of the fused-silica windows of the gas cell. On the other hand, the throughput for the hollow fiber chamber was calculated to be $50 \%$ and $67 \%$ for P1 and P2, respectively. The smaller throughput for the hollow fiber may be due to coupling losses at the entrance of the hollow fiber and the linear propagation losses inside the fiber. For propagation of the $\mathrm{EH}_{11}$ mode inside the hollow fiber, the linear propagation loss through the hollow fiber is calculated to be $9 \%$ at $1200 \mathrm{~nm}$ and $4 \%$ at $800 \mathrm{~nm}$ [27]. By assuming a coupling into the $\mathrm{EH}_{11}$ mode and propagation of the mode inside the hollow fiber, the efficiency of coupling at the entrance of the hollow fiber is calculated to be $61 \%$ and $78 \%$ for P1 and P2, respectively. The smaller coupling efficiency for P1 than P2 arises from the too large beam diameter of $\mathrm{P} 1(300 \mu \mathrm{m})$ to be perfectly coupled into the hollow fiber.

Table 1. Input and output energies of $\mathrm{P} 1$ and $\mathrm{P} 2$.

\begin{tabular}{cccccc}
\hline & \multicolumn{2}{c}{ Gas Cell } & & \multicolumn{2}{c}{ Hollow Fiber } \\
\cline { 2 - 3 } \cline { 5 - 6 } & Input & Output & & Input & Output \\
\hline $\mathrm{P} 1,1200 \mathrm{~nm}$ & $262 \mu \mathrm{J}$ & $236 \mu \mathrm{J}$ & & $278 \mu \mathrm{J}$ & $140 \mu \mathrm{J}$ \\
$\mathrm{P} 2,800 \mathrm{~nm}$ & $245 \mu \mathrm{J}$ & $220 \mu \mathrm{J}$ & & $252 \mu \mathrm{J}$ & $168 \mu \mathrm{J}$ \\
\hline
\end{tabular}

The pulse durations of the input pulses were measured based on cross-correlation frequency-resolved optical gating, which allows for the characterization of the two unknown pulses simultaneously [28]. The pulse durations obtained were 50-60 fs.

The spectra of the output beams measured at different pressures for both the gas cell and the hollow fiber chamber are shown in Figure 2. The efficiency in the generation of the anti-Stokes emission increased with increasing gas pressure for both cases. The spectral width of the emission increased because of SPM and XPM induced by the intense input pump pulses $[9,10,12,14]$. In the case of the gas cell, the anti-Stokes emission lines were well separated from each other in the spectrum at pressures ranging up to $2 \mathrm{~atm}$, while the spectrum became continuous above $1 \mathrm{~atm}$ in the case of the hollow fiber. The difference could be explained by the longer interaction length in the hollow fiber than in the gas cell. The spectrum of the output beam from the hollow fiber became a continuum at $2 \mathrm{~atm}$, as shown in Figure 3. Even at a pressure of $0.8 \mathrm{~atm}$, the influence of the phase modulation was appreciable in the case of the hollow fiber. An image of the output beam from the hollow fiber is shown in Figure 4, which was taken after passing the beam through a fused-silica prism and projecting the separated beam on a white screen. The spots are not separated from each other because of the broad bandwidth of the emission induced by the phase modulations. The spectral width of the anti-Stokes emission generated in the hollow fiber supported a sub-10 fs pulse duration, even at a pressure of 1 atm; no spectral overlap between adjacent anti-Stokes emission features was observed. Similar results were reported by Sali et al., in which these authors used longer pump pulses emitting at shorter 
wavelengths than in this work [12]. The hollow fiber thus may be able to generate sub-10 fs multicolor laser pulses covering a wavelength region from the DUV to NIR by relying on proper dispersion compensation for each emission feature.

Figure 2. The spectra of output beams at different hydrogen pressures. (a) Gas cell; (b) Hollow fiber.

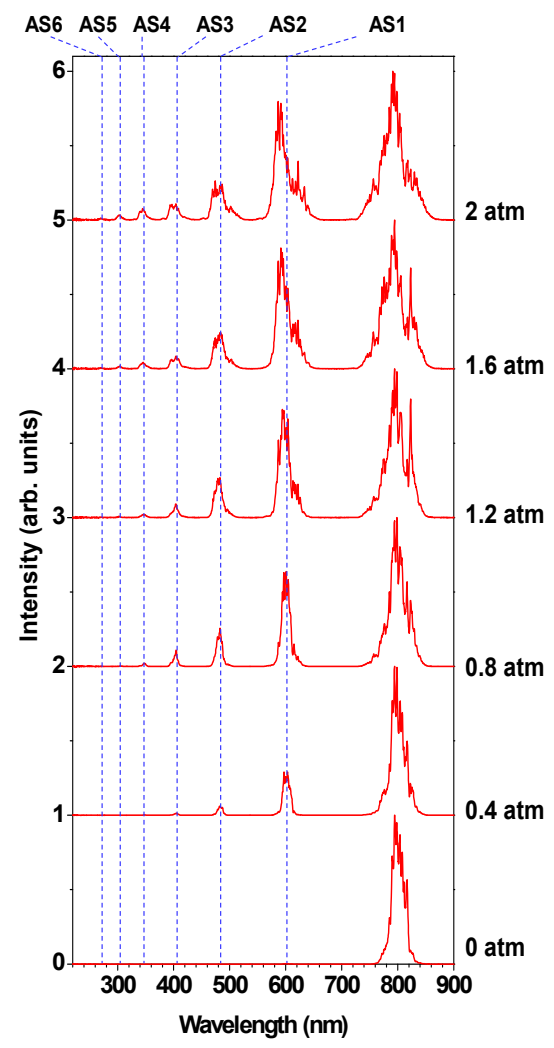

(a)

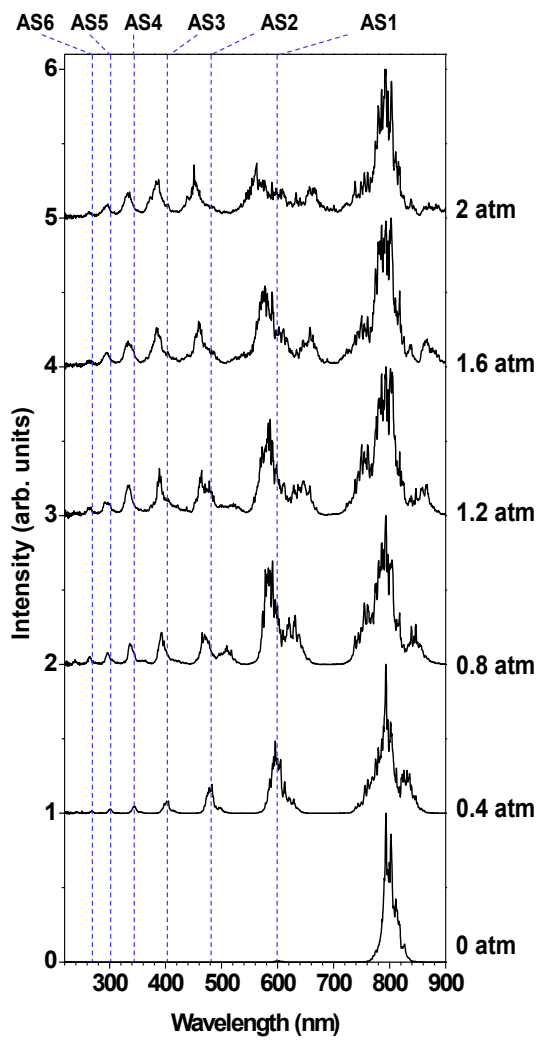

(b)

Figure 3. Spectra of output beams from the hollow fiber (black solid line) and the gas cell (red solid line) at a pressure of $2 \mathrm{~atm}$. The spectra for P1 are not shown in the figure.

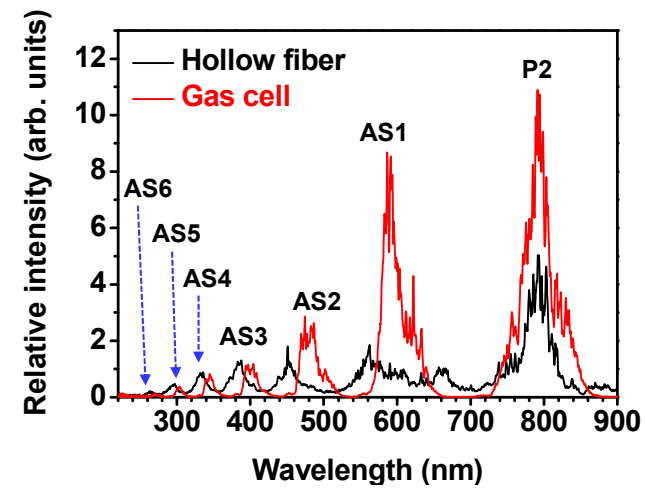

Figure 4. Photograph of the multicolor emission.

\begin{tabular}{|c|c|c|c|c|c|c|c|c|}
\hline AS8 & AS7 & AS6 & AS5 & AS4 & AS3 & AS2 & AS1 & $\mathbf{P 2}$ \\
\hline $\begin{array}{c}1 \\
220\end{array}$ & 241 & $\begin{array}{c}1 \\
267\end{array}$ & 300 & $\begin{array}{c}1 \\
343\end{array}$ & 401 & 481 & 600 & $\frac{1}{800 \mathrm{~nm}}$ \\
\hline
\end{tabular}


Although the efficiency of the XPM is smaller and the spectral width of anti-Stokes emission is narrower for the gas cell, the spectral widths of the multicolor emission achieved for the gas cell at a pressure of $2 \mathrm{~atm}$ support transform-limited pulse durations shorter than $15 \mathrm{fs}$. The spectral widths of the emission were $1200 \mathrm{~cm}^{-1}$ (AS1), $1300 \mathrm{~cm}^{-1}$ (AS2), $1200 \mathrm{~cm}^{-1}$ (AS3), and $1000 \mathrm{~cm}^{-1}$ (AS4). Similar to the case of the hollow fiber, the generation of sub-20 fs multicolor pulses may be possible after appropriate dispersion compensation.

\subsection{Energies of the Multicolor Emission}

For estimation of the energy of the anti-Stokes emission, the gas cell was first evacuated. Only P2 was focused into the gas cell and the pulse energy of P2 emerging from the cell was measured using a power meter. The spectrum of the output P2 was measured using a sensitivity-calibrated spectrometer, the position of which was kept unchanged during the subsequent experimental procedure. The measured pulse energy was divided by the integral of the spectral intensity of P2. The resulting value is hereafter referred to as the calibration factor. The gas cell was then filled with hydrogen gas and both P1 and P2 were focused into the gas cell. The spectrum of the output beam from the cell was measured using the spectrometer at different gas pressures. The integral of the spectral intensity for each anti-Stokes emission was multiplied by the calibration factor to estimate the pulse energy of the emission. The same procedure was repeated for the hydrogen-filled hollow fiber. Because of the nearly continuous structure of the spectrum observed at pressures higher than $1 \mathrm{~atm}$, the pulse energy could not be estimated for the hydrogen-filled hollow fiber at high pressures.

The results of the energy estimation are shown in Figure 5. In both the experiments using the gas cell and the hollow fiber, the energy of P2 was notably depleted by the generation of the anti-Stokes emission. The energies of the low-order anti-Stokes emission, i.e., AS1 and AS2, generated in the gas cell were 1.8 times higher than those generated in the hollow fiber. This result indicates that the gas cell is superior to the gas-filled hollow fiber in generating high-energy visible pulses. The conversion efficiency from P2 into the anti-Stokes emission was similar in these two cases (see the observed conversion efficiencies shown in Figure 5). This fact suggests that if there are no propagation and coupling losses for the hollow fiber, the anti-Stokes emission of AS1 and AS2 would have energies close to those for the gas cell. The energies of the higher-order anti-Stokes emission, on the other hand, were larger for the hollow fiber than those for the gas cell. The conversion efficiencies from P2 to the anti-Stokes emission of AS5 and AS6 were three times and four times larger than those generated in the gas cell, respectively, as shown in Figure 5c,f.

The energies of P1 and P2 for the gas cell were reduced until the output energies were the same as those in the hollow fiber experiment ( $140 \mu \mathrm{J}$ for P1 and $160 \mu \mathrm{J}$ for P2). For the energy reduction, the neutral density filters placed in the input beam paths were employed (see Figure 1). The filters with thicknesses of $2 \mathrm{~mm}$ would not have stretched the pump pulses appreciably; each filter stretches a 35 -fs pulse at $800 \mathrm{~nm}$ by only $0.5 \mathrm{fs}$. The resultant spectrum of the output beam from the gas cell pressurized at $2 \mathrm{~atm}$ is shown in Figure 6, together with the spectrum measured using the hollow fiber without a reduction in the pump energy. No notable difference was observed in the energies of the first- and second-order anti-Stokes emission under these conditions, although the spectral intensities of the emission were higher for the gas cell. This result suggests that the pulse energy of the visible 
emission from the hollow fiber does not exceed that from the gas cell, even under the ideal condition that there are no propagation and coupling losses for the hollow fiber.

Figure 5. The pressure dependence of the energy for the output beams. (a-c) The gas cell and $(\mathbf{d}-\mathbf{f})$ the hollow fiber. The maximum energy obtained for each anti-Stokes emission is indicated together with the conversion efficiency from P2 to the anti-Stokes emission. For $\mathrm{P} 2$, the ratio of the output energy measured at a specified pressure and the output energy measured at $0 \mathrm{~atm}$ is listed in each panel.
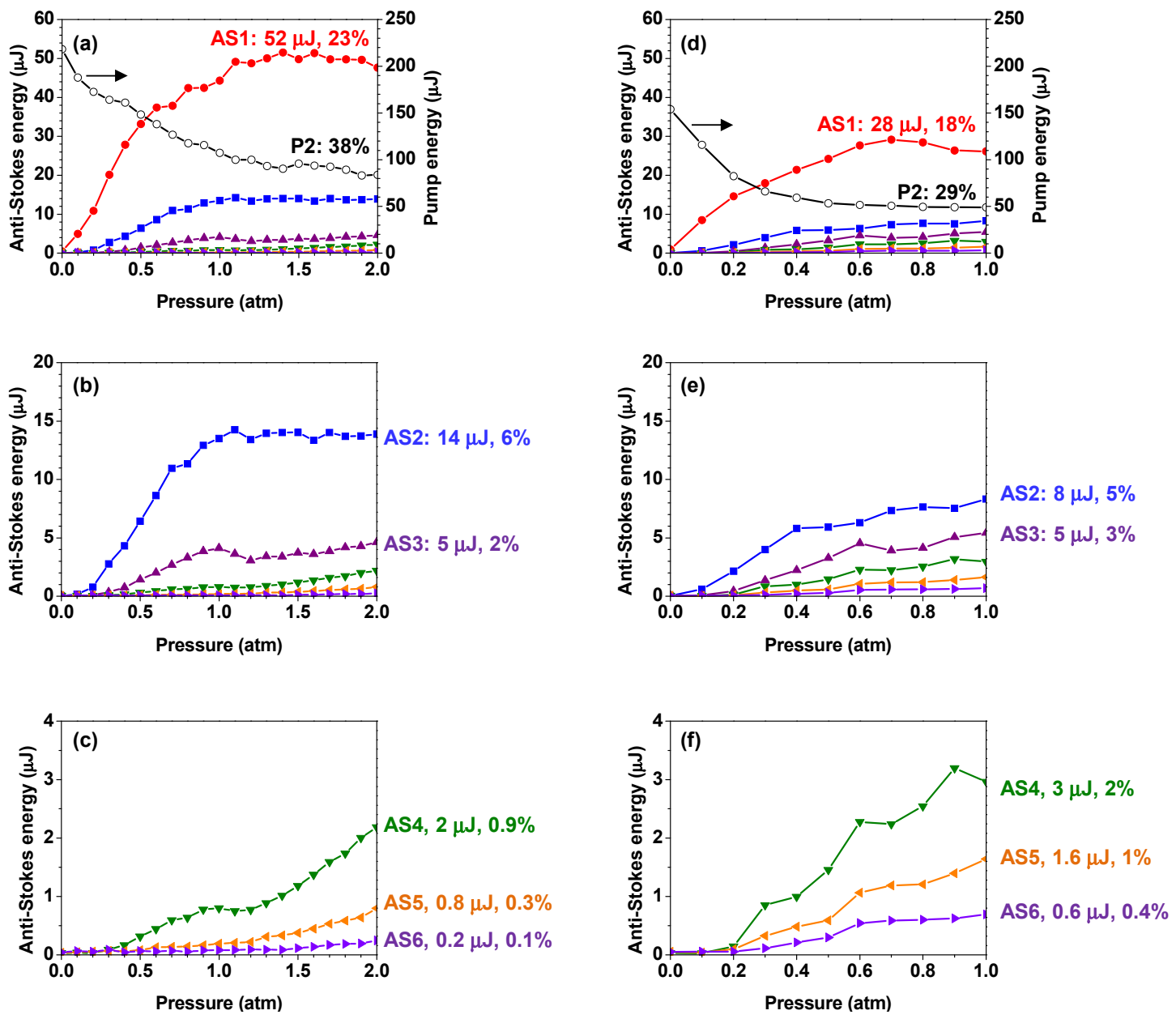

Figure 6. Spectra of the output beam from the gas cell (red solid line) and the hollow fiber (black solid line) measured at a pressure of $2 \mathrm{~atm}$. The energies of the input pump pulses for the gas cell are lower than those for the hollow fiber.

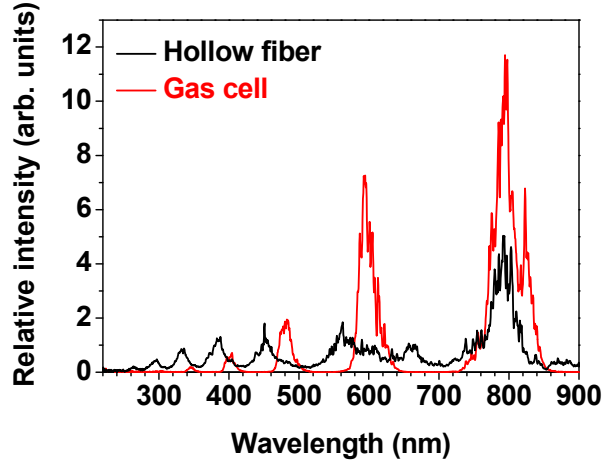




\subsection{Four-Wave Mixing}

The efficiency in FWM is determined by parameters such as the intensities of the pump pulses, phase mismatch, and the interaction length. Under the assumption that no depletion occurs for pump pulses, the intensity of the first-order anti-Stokes emission, $I_{\mathrm{AS} 1}$, can be described as $[29,30]$ :

$$
I_{\mathrm{AS} 1}=\frac{\epsilon_{0} n \omega_{\mathrm{AS} 1}{ }^{2} n_{2}{ }^{2} z^{2}}{2 c}\left|\varepsilon_{\mathrm{P} 1}\right|^{2}\left|\varepsilon_{\mathrm{P} 2}\right|^{4} \sin c^{2}\left(-\frac{\Delta \beta z}{2}\right)
$$

where $\epsilon_{0}, c, n, n_{2}, z$, and $\omega_{\mathrm{AS} 1}$, are the vacuum permittivity, the velocity of light in vacuum, the linear and nonlinear refractive indices of the gas, the interaction length, and the angular frequency of AS1, respectively. The intensities of $\mathrm{P} 1\left(I_{\mathrm{P} 1}\right)$ and $\mathrm{P} 2\left(I_{\mathrm{P} 2}\right)$ are proportional to the square of the corresponding electric field amplitudes, $\varepsilon_{\mathrm{P} 1}$ and $\varepsilon_{\mathrm{P} 2}$, respectively. The phase mismatch $\Delta \beta$ in the equation is expressed as $\beta_{\mathrm{P} 1}+\beta_{\mathrm{AS} 1}-2 \beta_{\mathrm{P} 2}$, where $\beta_{\mathrm{P} 1}, \beta_{\mathrm{AS} 1}$, and $\beta_{\mathrm{P} 2}$ stand for the propagation constants for $\mathrm{P} 1, \mathrm{AS} 1$, and $\mathrm{P} 2$. The phase mismatch in the hollow fiber can be calculated by taking into account the contributions of the gas and the waveguide [27,29,30].

In the generation of multicolor beams through cascaded FWM, the anti-Stokes emission is generated in the first step and higher-order anti-Stokes emission is then generated during the propagation in the gas. The energy of the first anti-Stokes emission is higher than that of the high-order anti-Stokes emission and it is a good measure of the efficiency of the multicolor generation.

Figure 7. Spectra of the output beam from the hollow fiber at $0.4 \mathrm{~atm}$ (black solid line) and $0.5 \mathrm{~atm}$ (blue solid line) and the gas cell (red solid line) at $2 \mathrm{~atm}$. The spectrum is normalized by the intensity of the highest peak.

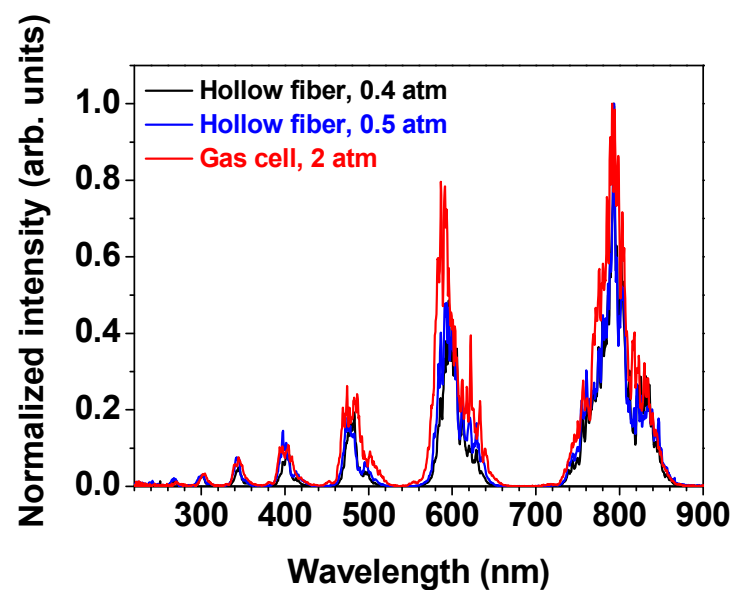

Equation (1) can be used to calculate the gas pressure in the hollow fiber, which leads to a similar efficiency of multicolor generation as that in the gas cell with a pressure of $2 \mathrm{~atm}$. The value of $\Delta \beta z / 2$ in Equation (1) is calculated to be $0.66 \mathrm{rad}$ for the gas cell and hence the value of $\sin c^{2}(-\Delta \beta z / 2)$ can be approximated as unity. From Equation (1) and the fact that the nonlinear refractive index is proportional to the gas pressure, the conversion efficiency from the pump to the first-order anti-Stokes emission, $I_{\mathrm{AS} 1} / I_{\mathrm{P} 2}$, is proportional to $I_{\mathrm{P} 1} I_{\mathrm{P} 2} p^{2} z^{2}$, where $p$ is the gas pressure. The output energies of $\mathrm{P} 1$ and $\mathrm{P} 2$ in the case of the hollow fiber were 0.59 and 0.76 times smaller than those for the gas cell, respectively, as indicated in Table 1 . The value of $I_{\mathrm{P} 1} I_{\mathrm{P} 2}$ for the hollow fiber is hence considered to be 0.43 times smaller than that for the gas cell. From this fact, together with the ratio of the interaction 
lengths in the hollow fiber and the gas cell $(600 \mathrm{~mm} / 80 \mathrm{~mm})$, the gas pressure in the hollow fiber, which provides the same value of $I_{\mathrm{AS} 1} / I_{\mathrm{P} 2}$ as for the gas cell, is calculated to be $0.4 \mathrm{~atm}$. In Figure 7 , we show the spectra of the output beams from the hollow fiber at 0.4 and 0.5 atm and the gas cell at $2 \mathrm{~atm}$ for comparison. Excellent agreement is observed between the spectra for the hollow fiber at 0.4-0.5 atm and for the gas cell at 2 atm, although no pump depletion is assumed to occur in the model used in Equation (1).

\subsection{Phase Mismatch in the Generation of High-Order Anti-Stokes Emission}

In Table 2, the values of $\Delta \beta z / 2$ in the generation of anti-Stokes emission are shown for the pathways with the energy conservations of $-\omega_{\mathrm{P} 1}+\omega_{\mathrm{P} 2}+\omega_{\mathrm{ASN}-1}-\omega_{\mathrm{ASN}}=0$, where $\omega_{\mathrm{P} 1}, \omega_{\mathrm{P} 2}, \omega_{\mathrm{ASN}-1}$, and $\omega_{\mathrm{ASN}}$ are the angular frequencies of $\mathrm{P} 1, \mathrm{P} 2,(N-1)^{\text {th }}$-order anti-Stokes, and $N^{\text {th }}$-order anti-Stokes emission. In the calculation, the propagation length is assumed to be $600 \mathrm{~mm}$ for the hollow fiber and $80 \mathrm{~mm}$ for the gas cell and the gas pressures are assumed to be $1 \mathrm{~atm}$ and $2 \mathrm{~atm}$, respectively. The value of $\Delta \beta z / 2$ for AS1 is lower than $\pi$ in the both cases of the gas cell and hollow fiber. As shown in Figure 5, the conversion efficiency from P2 to AS1 saturated at pressures higher than $1 \mathrm{~atm}$ and $0.5 \mathrm{~atm}$ for the gas cell and the hollow fiber, respectively. The saturated conversion efficiency from P2 to AS1 was similar in both cases $(23 \%$ for the gas cell and $18 \%$ for the hollow fiber). From these facts, the saturation would be related to the consumption of the energy for the generation of high-order anti-Stokes emission rather than the phase mismatch. In other words, the waveguide dispersion of the hollow fiber does not have notable influence to the conversion efficiency from P2 to AS1. Change in the parameters of the hollow fiber, such as use of a longer hollow fiber and a hollow fiber with a smaller core diameter, therefore, would not lead to a higher conversion efficiency to AS1. It just shifts the saturation pressure to a lower pressure. The energy of the visible multi-color emission (AS1) emitted from a gas cell is therefore expected to be always higher than that from a hollow fiber.

Table 2. Calculated value of $\Delta \beta z / 2$ for anti-Stokes emission.

\begin{tabular}{ccccccc}
\hline & \multicolumn{7}{c}{$\Delta \beta \boldsymbol{z} / \mathbf{2}$ (rad) } \\
\cline { 2 - 7 } & AS1 & AS2 & AS3 & AS4 & AS5 & AS6 \\
\hline Hollow fiber, 1 atm & 1.39 & 4.36 & 8.61 & 14.14 & 21.15 & 29.89 \\
Gas cell, 2 atm & 0.66 & 1.57 & 2.75 & 4.26 & 6.15 & 8.49 \\
\hline
\end{tabular}

The values of the $\Delta \beta z / 2$ are non-negligible for all the anti-Stokes emission and exceed $\pi$, except AS1 for the hollow fiber and except AS1, AS2, and AS3 for the gas cell. This fact does not explain the increase in the energy of the high-order anti-Stokes emission with increasing gas pressure, which was observed in the experiment (Figure 5c). For high-order anti-Stokes emission, other pathways therefore need to be considered.

There are several possible pathways useful for the generation of the high-order anti-Stokes emission. For instance, four paths- $-\omega_{\mathrm{P} 1}+\omega_{\mathrm{P} 2}+\omega_{\mathrm{AS} 3}-\omega_{\mathrm{AS} 4}=0,-\omega_{\mathrm{P} 2}+\omega_{\mathrm{AS} 1}+\omega_{\mathrm{AS} 3}-\omega_{\mathrm{AS} 4}=0$, $-\omega_{\mathrm{AS} 1}+\omega_{\mathrm{AS} 2}+\omega_{\mathrm{AS} 3}-\omega_{\mathrm{AS} 4}=0$, and $-\omega_{\mathrm{AS} 2}+\omega_{\mathrm{AS} 3}+\omega_{\mathrm{AS} 3}-\omega_{\mathrm{AS} 4}=0$-would contribute to the generation of the fourth-order anti-Stokes emission, AS4. Among them, the phase mismatch, $\Delta \beta$, is the highest for the first pathway and lowest for the fourth pathway. $\Delta \beta z / 2$ is calculated to be $1.5 \mathrm{rad}$ for the fourth path in the case of the gas cell with a pressure of $2 \mathrm{~atm}$. This value is lower than $\pi / 2$ and the 
path would contribute to the increase in the anti-Stokes signal intensity at high pressures. Since the degree of phase mismatch is proportional to the gas pressure, the path most effective for the generation of the Raman emission would change depending on the gas pressure, even under the same experimental conditions for the propagation length and the input pulse intensities. This effect may be of importance, particularly in the DUV, at which numerous possible pathways are plausible for the generation of Raman emission. For the gas-filled hollow fiber, it should be necessary to take into account the contributions from high-order propagation modes. For several combinations of high-order propagation modes, the gas pressure at which phase matching is satisfied becomes higher than the phase-matching gas pressure for the lowest-order propagation modes [29-32].

\subsection{Spectral Blueshift in the Ultraviolet Sidebands}

A spectral blueshift was observed for the anti-Stokes emission in the ultraviolet region when a hollow fiber was filled at pressures higher than $0.4 \mathrm{~atm}$. As can be seen in Figure $5 \mathrm{~b}$, the wavelengths of the emission are continuously blueshifted with increasing gas pressure. This blueshift was not observed for the gas cell at pressures up to $2 \mathrm{~atm}$. The blueshift can be explained in terms of the pulse chirp of each emission. In Figure 8, we calculate the group delay of each emission with respect to P2 for (a) the gas cell and (b) the hollow fiber under the propagation mode of $\mathrm{EH}_{11}$ after the propagation length of $z=80$ and $600 \mathrm{~mm}$, respectively. The results suggest that the group delay of the high-order anti-Stokes emission in the hollow fiber (AS4-6) significantly increases with an increase in gas pressure. The anti-Stokes emission delayed with respect to the pump pulses would temporally overlap at the trailing edges of the pump pulses and the phase could be modulated via XPM induced by the pump pulses, resulting in a spectral blueshift. On the other hand, the group delay is small in the gas cell for all orders of the anti-Stokes emission. Thus, the group delay could have a negligible effect on the frequency of the anti-Stokes emission in the case of the gas cell, as shown in the experimental results of Figure $2 \mathrm{a}$.

Figure 8. Group delay calculated for each emission against that of $\mathrm{P} 2$ for the gas cell (a) and the hollow fiber (b) at different gas pressures.

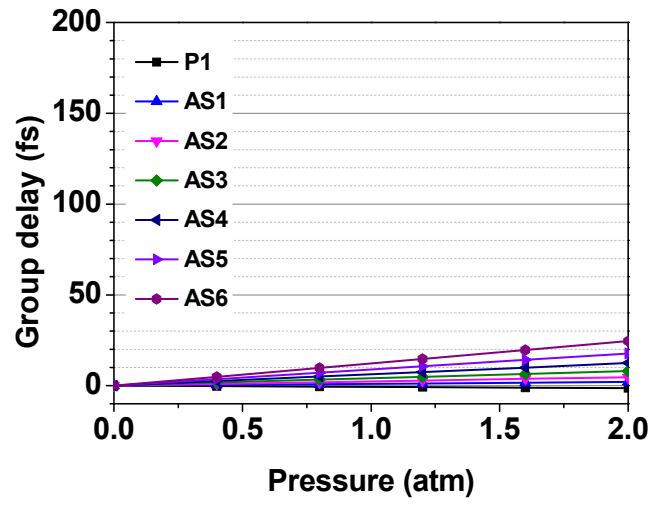

(a)

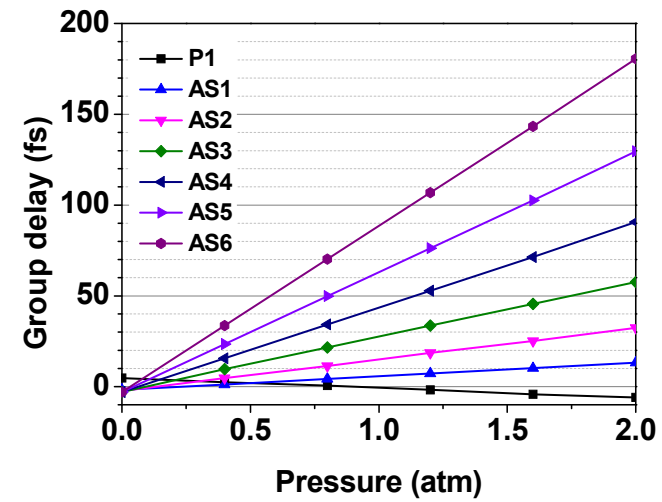

(b)

The group delays of AS4, AS5, and AS6 exceed the pulse durations of the pump pulses of 50 fs at pressures higher than $1.2 \mathrm{~atm}$ in the case of the hollow fiber (Figure 8b). These delays make the interaction between pump pulses and the high-order anti-Stokes pulses difficult for the generation of 
higher-order anti-Stokes emission via FWM. For this reason, combinations of frequency components with small phase mismatches for FWM should be effective for the generation of high-order anti-Stokes emission at high pressures for the hollow fiber (Figure 5f).

\section{Conclusions}

We have compared two approaches using a gas cell and a hollow fiber to generate high-energy multicolor fs pulses. The gas cell provides multicolor emission in the visible range with higher energies than those obtained using the hollow fiber. The pulse energies were several tens of microjoules, which were one order of magnitude larger than those obtained based on FWM in bulk media. Because of the simultaneous generation of SPM and XPM, the spectral bandwidth of the anti-Stokes emission increased with increasing gas pressure. The resultant bandwidth using the gas cell supports transform-limited pulse durations shorter than 15 fs. A broader bandwidth was obtained for multicolor emission generated in the gas-filled hollow fiber. In this case, multicolor laser pulses obtained in the DUV region had higher energies than pulses obtained using the gas cell, where the energy range was a few microjoules. The high-energy visible pulses generated in the gas cell are suitable for spectroscopic studies in the gas phase, such as multiphoton ionization of aromatic compounds and condensed-phase compounds. The ultraviolet pulses generated by the gas-filled hollow fiber, on the other hand, may be applied to spectroscopy of liquid and solid phases, such as the ultrafast transient absorption spectroscopy in biologically significant molecules [33].

\section{Acknowledgments}

This research was supported by the Japan Society for the Promotion of Science (JSPS) KAKENHI Grant No. 23245017.

\section{Author Contributions}

Drafting of manuscript: Kazuya Motoyoshi, Yuichiro Kida; Acquisition of data: Kazuya Motoyoshi; Analysis and interpretation of data: Kazuya Motoyoshi, Yuichiro Kida; Critical revision: Yuichiro Kida, Totaro Imasaka; Planning and supervision of the research: Yuichiro Kida, Totaro Imasaka.

\section{Conflicts of Interest}

One of the co-authors, Totaro Imasaka, is the guest editor of the special issue.

\section{References}

1. Imasaka, T.; Kawasaki, S.; Ishibashi, N. Generation of More than 40 Laser Emission Lines from the Ultraviolet to the Visible Regions by Two-Color Stimulated Raman Effect. Appl. Phys. B 1989, 49, 389-392.

2. Yavuz, D.; Walker, D.; Shverdin, M.; Yin, G.; Harris, S. Quasiperiodic Raman Technique for Ultrashort Pulse Generation. Phys. Rev. Lett. 2003, 91, 233602.

3. Shverdin, M.; Walker, D.; Yavuz, D.; Yin, G.; Harris, S. Generation of a Single-Cycle Optical Pulse. Phys. Rev. Lett. 2005, 94, 033904. 
4. Burzo, A.M.; Chugreev, A.V.; Sokolov, A.V. Optimized Control of Generation of Few Cycle Pulses by Molecular Modulation. Opt. Commun. 2006, 264, 454-462.

5. Chen, W.-J.; Hsieh, Z.-M.; Huang, S.; Su, H.-Y.; Lai, C.-J.; Tang, T.-T.; Lin, C.-H.; Lee, C.-K.; Pan, R.-P.; Pan, C.-L.; et al. Sub-Single-Cycle Optical Pulse Train with Constant Carrier Envelope Phase. Phys. Rev. Lett. 2008, 100, 5-8.

6. Yoshikawa, S.; Imasaka, T. A New Approach for the Generation of Ultrashort Optical Pulses. Opt. Commun. 1993, 96, 94-98.

7. Kaplan, A. Subfemtosecond Pulses in Mode-Locked $2 \pi$ Solitons of the Cascade Stimulated Raman Scattering. Phys. Rev. Lett. 1994, 73, 1243-1246.

8. Irie, Y.; Imasaka, T. Generation of Vibrational and Rotational Emissions by Four-Wave Raman Mixing Using an Ultraviolet Femtosecond Pump Beam. Opt. Lett. 1995, 20, 2072-2074.

9. Kawano, H.; Hirakawa, Y.; Imasaka, T. Generation of More than 40 Rotational Raman Lines by Picosecond and Femtosecond Ti:sapphire Laser for Fourier Synthesis. Appl. Phys. B 1997, 65, 1-4.

10. Kawano, H.; Hirakawa, Y.; Imasaka, T. Generation of High-Order Rotational Lines in Hydrogen by Four-Wave Raman Mixing in the Femtosecond Regime. IEEE J. Quantum Electron. 1998, 34, 260-268.

11. Crespo, H.; Mendonça, J.T.; Dos Santos, A. Cascaded Highly Nondegenerate Four-Wave-Mixing Phenomenon in Transparent Isotropic Condensed Media. Opt. Lett. 2000, 25, 829-831.

12. Sali, E.; Kinsler, P.; New, G.; Mendham, K.; Halfmann, T.; Tisch, J.; Marangos, J. Behavior of High-Order Stimulated Raman Scattering in a Highly Transient Regime. Phys. Rev. A 2005, $72,013813$.

13. Zhi, M.; Sokolov, A.V. Broadband Coherent Light Generation in a Raman-Active Crystal Driven by Two-Color Femtosecond Laser Pulses. Opt. Lett. 2007, 32, 2251-2253.

14. Turner, F.C.; Trottier, A.; Strickland, D.; Losev, L.L. Transient Multi-Frequency Raman Generation in $\mathrm{SF}_{6}$. Opt. Commun. 2007, 270, 419-423.

15. Liu, J.; Zhang, J.; Kobayashi, T. Broadband Coherent Anti-Stokes Raman Scattering Light Generation in BBO Crystal by Using Two Crossing Femtosecond Laser Pulses. Opt. Lett. 2008, 33, 1494-1496.

16. Liu, J.; Kobayashi, T. Generation of Sub-20-fs Multicolor Laser Pulses Using Cascaded Four-Wave Mixing with Chirped Incident Pulses. Opt. Lett. 2009, 34, 2402-2404.

17. Liu, J.; Kobayashi, T. Generation of $\mu \mathrm{J}$ Multicolor Femtosecond Laser Pulses Using Cascaded Four-Wave Mixing. Opt. Express 2009, 17, 4984-4990.

18. Weigand, R.; Mendonça, J.; Crespo, H. Cascaded Nondegenerate Four-Wave-Mixing Technique for High-Power Single-Cycle Pulse Synthesis in the Visible and Ultraviolet Ranges. Phys. Rev. A 2009, 79, 063838.

19. Silva, J.; Crespo, H.; Weigand, R. Generation of High-Energy Vacuum UV Femtosecond Pulses by Multiple-Beam Cascaded Four-Wave Mixing in a Transparent Solid. Appl. Opt. 2011, 50, 1968-1973.

20. Shitamichi, O.; Imasaka, T. High-Order Raman Sidebands Generated from the near-Infrared to Ultraviolet Region by Four-Wave Raman Mixing of Hydrogen Using an Ultrashort Two-Color Pump Beam. Opt. Express 2012, 20, 27959-27965. 
21. Cui, Z.; Chaturvedi, M.; Tian, B.; Ackert, J.; Turner, F.C.; Strickland, D. Spectral Red-Shifting of Multi-Frequency Raman Orders. Opt. Commun. 2013, 288, 118-121.

22. Kobayashi, T.; Saito, T.; Ohtani, H. Real-Time Spectroscopy of Transition States in Bacteriorhodopsin during Retinal Isomerization. Nature 2001, 414, 531-534.

23. Lanzani, G.; Cerullo, G.; Brabec, C.; Sariciftci, N. Time Domain Investigation of the Intrachain Vibrational Dynamics of a Prototypical Light-Emitting Conjugated Polymer. Phys. Rev. Lett. 2003, 90, 047402.

24. Yabushita, A.; Kobayashi, T. Primary Conformation Change in Bacteriorhodopsin on Photoexcitation. Biophys. J. 2009, 96, 1447-1461.

25. Rankin, B.R.; Kellner, R.R.; Hell, S.W. Stimulated-Emission-Depletion Microscopy with a Multicolor Stimulated-Raman-Scattering Light Source. Opt. Lett. 2008, 33, 2491-2493.

26. Stoicheff, B.P. High Resolution Raman Spectroscopy of Gases: IX. Spectra of $\mathrm{H}_{2}, \mathrm{HD}$, and $\mathrm{D}_{2}$. Can. J. Phys. 1957, 35, 730-741.

27. Marcatili, E.A.J.; Schmeltzer, R.A. Hollow Metallic and Dielectric Waveguides for Long Distance Optical Transmission and Lasers. Bell Syst. Tech. J. 1964, 43, 1783-1809.

28. Kida, Y.; Nakano, Y.; Motoyoshi, K.; Imasaka, T. Frequency-Resolved Optical Gating with Two Nonlinear Optical Processes. Opt. Lett. 2014, 39, 3006-3009.

29. Durfee, C.G.; Misoguti, L.; Backus, S.; Kapteyn, H.C.; Murnane, M.M. Phase Matching in Cascaded Third-Order Processes. J. Opt. Soc. Am. B 2002, 19, 822-831.

30. Kida, Y.; Imasaka, T. Optical Parametric Amplification of a Supercontinuum in a Gas. Appl. Phys. B 2013, doi:10.1007/s00340-013-5751-4.

31. Misoguti, L.; Backus, S.; Durfee, C.; Bartels, R.; Murnane, M.; Kapteyn, H. Generation of Broadband VUV Light Using Third-Order Cascaded Processes. Phys. Rev. Lett. 2001, 87, 013601.

32. Faccio, D.; Grün, A.; Bates, P.K.; Chalus, O.; Biegert, J. Optical Amplification in the near-Infrared in Gas-Filled Hollow-Core Fibers. Opt. Lett. 2009, 34, 2918-2920.

33. Kobayashi, T.; Kida, Y. Ultrafast Spectroscopy with Sub-10 fs Deep-Ultraviolet Pulses. Phys. Chem. Chem. Phys. 2012, 14, 6200-6210.

(C) 2014 by the authors; licensee MDPI, Basel, Switzerland. This article is an open access article distributed under the terms and conditions of the Creative Commons Attribution license (http://creativecommons.org/licenses/by/3.0/). 